\title{
Toward Right-Fidelity Rotorcraft Conceptual Design
}

\author{
Jeffrey D. Sinsay ${ }^{1}$ \\ Aeroflightdynamic Directorate (AMRDEC) \\ U.S. Army Research, Development, and Engineering Command
}

Wayne Johnson

Aeromechanics Branch

National Aeronautics and Space Adminstration

Ames Research Center

Moffett Field, CA

\author{
Abstract for $48^{\text {th }}$ AIAA Aerospace Scientists Meeting (CREATE-AV Special Session) \\ Orlando FL \\ January 4-7, 2010
}

The aviation Advanced Design Office (ADO) of the US Army Aeroflightdynamics Directorate (AMRDEC) performs conceptual design of advanced Vertical Takeoff and Landing (VTOL) concepts in support of the Army's development and acquisition of new aviation systems. In particular, ADO engages in system synthesis to assess the impact of new technologies and their application to satisfy emerging warfighter needs and requirements. Fundamental to ADO being successful in accomplishing its role, is the ability to evaluate a wide array of proposed air vehicle concepts, and independently synthesize new concepts to inform Army and DoD decision makers about the tradespace in which decisions will be made (Figure 1). ADO utilizes a conceptual design (CD) process in the execution of its role. Benefiting from colocation with NASA rotorcraft researchers at the Ames Research Center, ADO and NASA have engaged in a survey of the current rotorcraft PD practices and begun the process of improving those capabilities to enable effective design and development of the next generation of VTOL systems.

$\mathrm{A}$ unique aspect of $\mathrm{CD}$ in $\mathrm{ADO}$ is the fact that actual designs developed in-house are not intended to move forward in the development process. Rather, they are used as reference points in discussions about requirements development and technology impact. The ultimate products of $\mathrm{ADO} \mathrm{CD}$ efforts are technology impact assessments and specifications which guide industry design activity. The fact that both the requirement and design are variables in the tradespace adds to the complexity of the CD process. A frequent need is ability to assess the relative "cost" of variations in requirement for a diverse set of VTOL configurations. Each of these configurations may have fundamentally different response characteristics to this requirement variation, and such insight into how different requirements drive different designs is a critical insight $\mathrm{ADO}$ attempts to provide decision makers.

The processes and tools utilized are driven by the timeline in which questions must be answered. This can range from quick "back-of-the-envelope" assessments of a configuration made in an afternoon, to more detailed tradespace explorations that can take upwards of a year to complete. A variety of spreadsheet based tools and conceptual design codes are currently in use. The in-house developed conceptual sizing code RC (RotorCraft) has been the preferred tool of choice for CD activity for a number of years. Figure 2 illustrates the long standing coupling between RC and solid modeling tools for layout, as well as a number of ad-hoc interfaces with external analyses. RC contains a sizing routine that is built around the use of momentum theory for rotors, classic finite wing theory, a referred parameter engine model, and semi-emperical weight estimation techniques. These methods lend themselves to rapid solutions, measured in seconds and minutes. The successful use of RC, however requires careful consideration of model input parameters and judicious comparison with existing aircraft to avoid unjustified extrapolation of results. RC is in fact a legacy of a series of codes whose development started in the early 1970s, and is best suited to the study of conventional helicopters and XV-15 style tiltrotors. Other concepts have been analyzed with RC, but typically it became necessary to modify the source code and methods for each unique configuration. Recent activity has lead to the development of a new code, NASA

\footnotetext{
${ }^{1}$ Corresponding Author: jeffrey.sinsay@us.army.mil, Work: (650)604-6157
} 
Design and Analysis of Rotorcraft (NDARC). NDARC uses a similar level of analytical fidelity as RC, but is built on a new framework intended to improve modularity and ability to rapidly model a wider array of concepts. Critical to achieving this capability is the decomposition of the aircraft system into a series of fundamental components which can then be assembled to form a wide-array of configurations. The paper will provide an overview of NDARC and its capabilities.

For more in-depth $\mathrm{CD}$ activities $\mathrm{ADO}$ has turned to higher fidelity analysis to enhance understanding of configurations, and anchor design points in the tradespace being explored in RC/NDARC. These activities begin to blur the traditional boundary between conceptual and preliminary design. These efforts initially focused on the use of comprehensive rotorcraft analysis tools, such as CAMRAD II, but more recently have explored interfacing with CFD and pilot-in-the-loop simulation tools. The interfaces between tools have suffered from an ad-hoc approach with multiple humans in-the-loop. This has resulted in slow turn-around and difficulty in keeping multiple models consistent. The requirements for a robust, modular information manager will be discussed.

Recently particular effort was invested by ADO in the solid-model-to-CFD interface for a tiltrotor, as part of the DoD's CREATE-AV program (see Figure 3), the results of which will be reported in this paper. The move from the simple representation of a tiltrotor necessary for layout work in support of CD to an OML suitable for analysis in CFD presented several key challenges that highlight the larger issue of how to utilize higher fidelity analyses in the $\mathrm{CD}$ process. The use of solid models to aid in visualization and layout work has been standard practice within ADO for over a decade. Previous to the use of CFD, the exact coordinates of the outer mold line were not of great importance, only the overall dimensions mattered. The use of high fidelity $\mathrm{CFD}$, however, requires the $\mathrm{CD}$ process to produce a high fidelity outer mold line to get reasonable predictions of the aerodynamic forces. This introduces a sub-design problem to the $\mathrm{CD}$ process that was not required previously when doing aircraft synthesis and sizing only with $\mathrm{RC} / \mathrm{NDARC}$. The introduction of these additional design tasks is a characteristic of the use of higherfidelity analysis in $\mathrm{CD}$.

It is this characteristic, of added design fidelity to go along with analysis fidelity, which poses the greatest challenge to improving the processes ADO uses in conceptual design. The level of personnel and financial resources, and required timeliness associated with conceptual design is not likely to dramatically change. Yet, as we look to squeeze greater performance out of air vehicles, write smarter specifications, and differentiate a wider array of viable concepts, it becomes critical that ADO find a way to utilize higherfidelity tools. The concept of "right-fidelity" becomes very important in considering the future evolution of $\mathrm{CD}$ processes. There is little value and unacceptable costs associated with defining conceptual designs to a level of detail greater than need to differentiate between them, or assess technical risk/pay-off. The driving choice in analysis fidelity should therefore not be necessarily the highest fidelity that can be computationally afforded, but rather the minimum level of fidelity needed to reach an acceptable level of uncertainty. This right-fidelity will likely vary across disciplines, but care must be exercised and tools created to ensure that model consistency is maintained regardless of the fidelity utilized.

There will likely be a gap between the maximum level of design fidelity that can be reasonably afforded and the minimum level required to achieve the desired level of certainty. The use of semiautomated design techniques, such as model parameterization and shape optimization can help narrow this gap. The use of system optimization techniques, while prolific in academic design activities has yet to be fully embraced at the system level in large-scale aerospace applications. For ADO, system optimization presents particular challenges. The primary goal of our work can be viewed as the definition of a relevant objective function that captures the diverse set of needs and requirements common to modern multi-role, multi-service aircraft.

Considering the aforementioned items, the paper will attempt to lay out a path forward for ADO that enables a greater use of right-fidelity analysis in conceptual design. A framework for moving forward will be established, and areas requiring further development will be identified. 


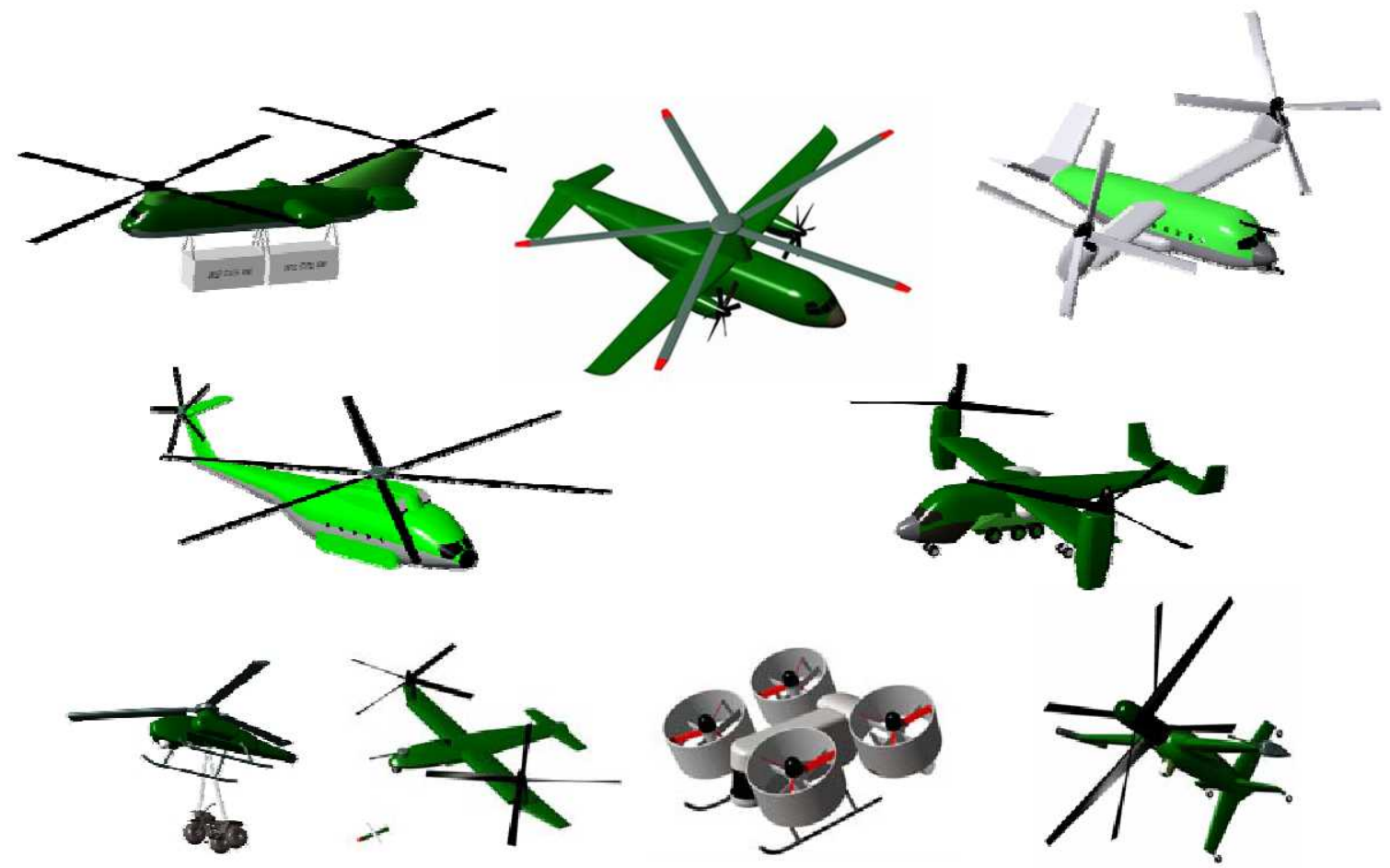

Figure 1 A diverse array of concepts and critical physical phenomoenon is a key characteristic of VTOL conceptual design.

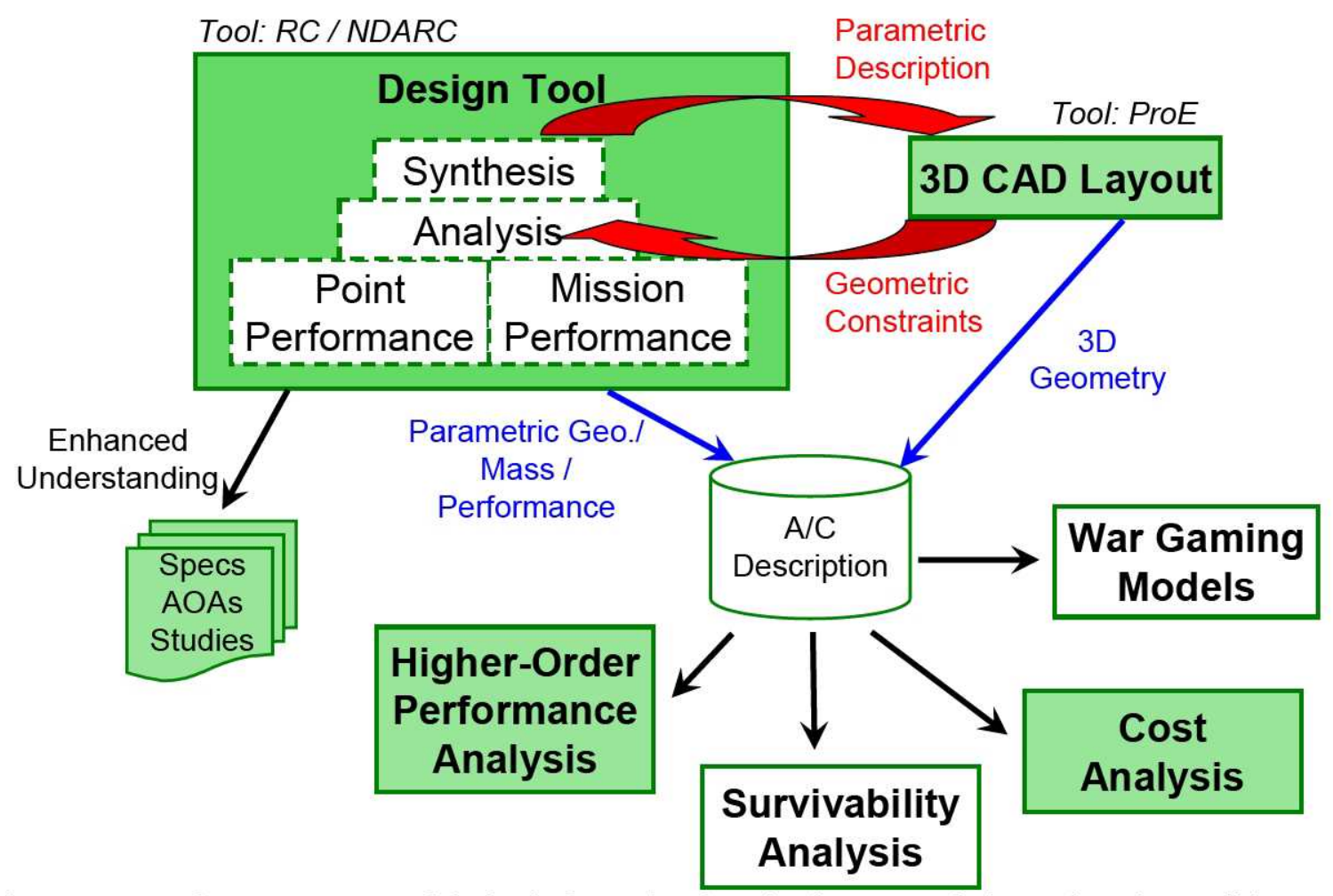

Figure 2 Currently ADO conceptual design is focused on iteration between a design code and 3D solid modeling tool to develop feasible concepts. Additional analysis can be performed via manual ad-hoc interfaces. 


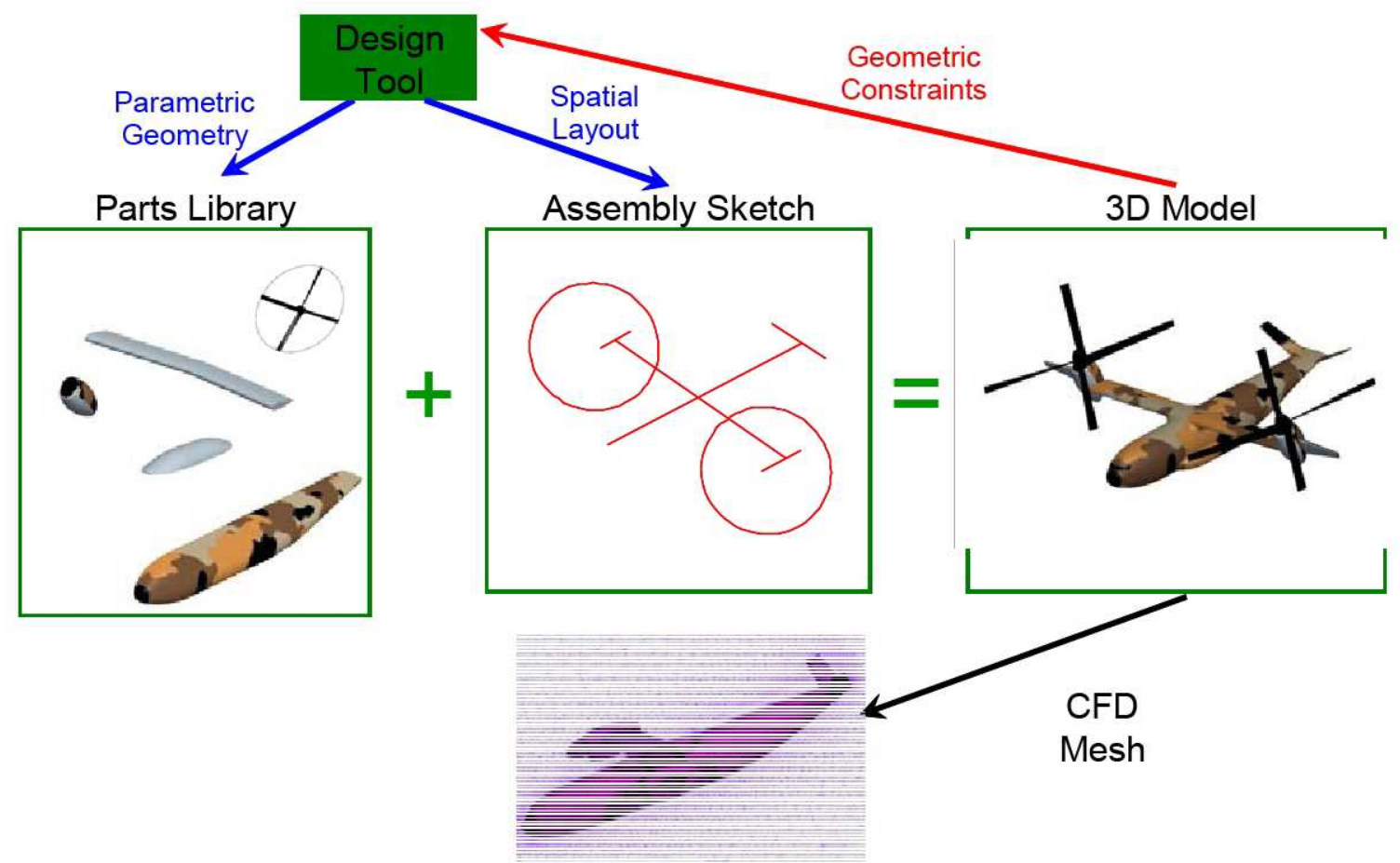

Figure 3 Using high level parametric data from the design code, parametric 3D solid models have been developed based on a library of primitive parts. These models can then be the basis for CFD mesh generation. 